\title{
3D microwave cavity with magnetic flux control and enhanced quality factor
}

\author{
Yarema Reshitnyk ${ }^{1}$, Markus Jerger ${ }^{2}$ and Arkady Fedorov ${ }^{1,2^{*}}$
}

\author{
"Correspondence: \\ a.fedorov@uq.edu.au \\ 'School of Mathematics and \\ Physics, The University of \\ Queensland, St Lucia, 4072, Australia \\ ${ }^{2} \mathrm{ARC}$ Centre of Excellence for \\ Engineered Quantum Systems, The \\ University of Queensland, 4072, St \\ Lucia, Australia
}

\begin{abstract}
Three-dimensional (3D) microwave cavities have been extensively used for coupling and interacting with superconducting quantum bits (qubits), providing a versatile platform for quantum control experiments and for realizing hybrid quantum systems. While having high quality factors $\left(>10^{6}\right)$ superconducting cavities do not permit magnetic field control of qubits. In contrast, cavities made of normal metals are transparent to magnetic fields, but experience lower quality factors $\left(\sim 10^{4}\right)$. We have created a hybrid cavity which is primarily composed of aluminium but also contains a small copper insert reaching the internal quality factor of $\simeq 10^{5}$, an order of magnitude improvement over all previously tested normal metal cavities. In order to demonstrate precise magnetic control, we performed spectroscopy of three superconducting qubits, where individual control of each qubit's frequency was exerted with small external wire coils. An improvement in quality factor and magnetic field control makes this 3D hybrid cavity an attractive new element for circuit quantum electrodynamics experiments.
\end{abstract}

Keywords: circuit QED; superconducting qubit; 3D microwave cavity; magnetic control

\section{Introduction}

Three-dimensional (3D) cavities with embedded superconducting qubits [1] have become one of the main platforms for circuit quantum electrodynamics experiments [2,3], and are also being used in conjunction with other quantum systems [4-6]. Within this architecture, the coherence of superconducting qubits has drastically improved, which shed light on the origin of coherence limitations [7]. Another advantage of this architecture is high internal quality factors (Q's) of 3D cavities. The quality factor can reach as much as $\sim 10^{9}$ (see Ref. [8] and references therein) and Q's of the order of $10^{6}$ can be reached without special attention to manufacturing and material purity. Superconducting 3D cavities are not without drawbacks, however. The walls of superconductive cavities perfectly screen the interior of the cavity from external magnetic fields, preventing flux control of the embedded qubits. Thermalization of the embedded devices is difficult, since superconductors, unlike normal metals, are bad thermal conductors. The common way around of these issues is to use cavities made from normal metal instead, but these have substantially smaller Q's of the order of 10,000 [9]. More sophisticated measures to reduce losses and thermalize the cavity walls were also investigated [10]. In addition, tunability of a superconducting

(c) 2016 Reshitnyk et al. This article is distributed under the terms of the Creative Commons Attribution 4.0 International License (http://creativecommons.org/licenses/by/4.0/), which permits unrestricted use, distribution, and reproduction in any medium, provided you give appropriate credit to the original author(s) and the source, provide a link to the Creative Commons license, and indicate if changes were made. 
qubit in a fully superconducting cavity has been realized [11] by having a part of the chip with the SQUID loop of the qubit outside of the cavity. While this method allows to retain high quality factor of the cavity it requires a specific design of the qubit and might not be well-suited for hybrid quantum systems.

\section{Results}

In this work we present measurements completed in a 3D microwave cavity of rectangular geometry with dimensions $30.00 \mathrm{~mm} \times 4.60 \mathrm{~mm} \times 27.40 \mathrm{~mm}$. The majority of this cavity was machined out of standard aluminium (alloy 6061) with a small insert machined out of oxygen free copper (C10100) as shown in Figure 1(b). The cavity has two microwave ports which can be used for either transmission or reflection measurements. Transmission measurements were used to perform spectroscopy and time-domain characterization of superconducting qubits placed inside the cavity. The internal $\mathrm{Q}$ of the cavity was determined by measuring the bare cavity in reflection, with the second port disconnected. All room-temperature measurements were performed with a vector network analyzer (VNA). Low temperature measurements were done in a dilution refrigerator with use of additional filtering of the microwave lines, a low-noise HEMT amplifier to amplify

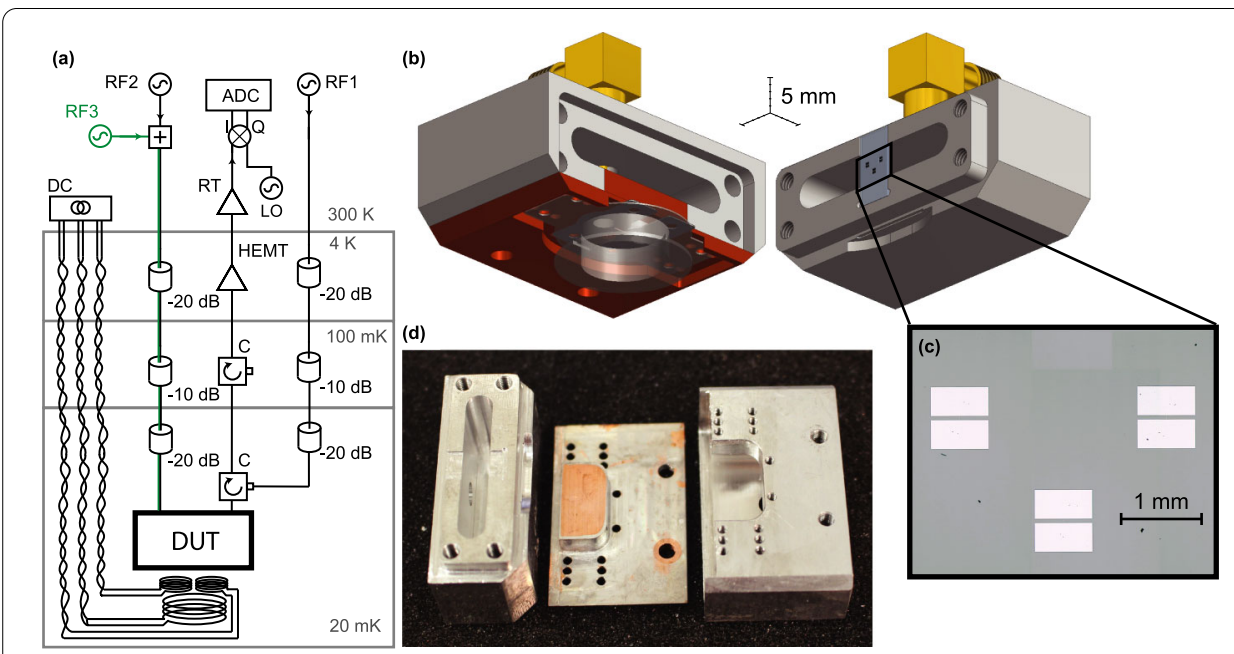

Figure 1 Measurement setup and device description. (a) Simplified diagram of the measurement setup at Millikelvin temperatures. The device under test (DUT) is a microwave cavity which properties are tested either by transmission or reflection measurements from its ports. To perform reflection measurements, a microwave tone (RF1) is applied to the output port of the cavity via a circulator (C). The reflected signal is amplified by a high-electron-mobility transistor (HEMT) amplifier at $4 \mathrm{~K}$ and a chain of room temperature (RT) amplifiers. The sample sits at $20 \mathrm{mK}$ and is isolated from the higher temperature stages by an additional circulator in series. The amplified signal is down-converted to an intermediate frequency of $25 \mathrm{MHz}$ in an IQ mixer, driven by a dedicated LO, and is digitized by an analog-to-digital converter (ADC) for data analysis. To perform transmission measurement a microwave signal (RF2) is applied to the input port of the cavity. For spectroscopy of the qubits, an additional excitation microwave tone (RF3) is applied together with the measurement signal. The magnetic field inside the cavity is controlled by three coils attached to the exterior of the cavity and wired to individual current sources (DC) at room temperature. (b) 3D model of the cavity with a chip containing three flux tunable transmon qubits. Most of the cavity body is made of Al (gray) with a Cu insert (brown). The Cu insert allows magnetic field from the three mounted coils to penetrate the interior of the cavity for individual control of all qubits frequencies. In addition, the Cu insert provides a beneficial thermal link to the chip. (c) Magnified image of the three transmon qubits on the chip. Each qubit consists of two Al capacitor plates connected via a line interrupted by a micron size DC SQUID (not visible at this scale). (d) Photograph of the cavity parts. To reduce losses due to possible gaps between Cu and Al parts, $\sim 2 \mu \mathrm{m}$ of Al were evaporated on the surfaces of the $\mathrm{Cu}$ insert which get in contact with $\mathrm{Al}$. 
the transmitted/reflected signal, and circulators to isolate the cavity from any noise from the amplifier and also to separate incoming and reflected signals when measuring in reflection (see Figure 1(a)).

We first performed a characterization of the cavity without superconducting qubits. The lowest resonant mode of the cavity (TE101) without a chip was found at $7.5905 \mathrm{GHz}$ at a temperature of $\sim 20 \mathrm{mK}$. The internal quality factors of the cavities with similar geometries made only out of $\mathrm{Cu}$ were found to be 4,800 at room temperature and 11,000 at $20 \mathrm{mK}$. The internal quality factor of aluminium cavity made of the same alloy were found 2,100 and $\simeq 450,000$ at room and milliKelvin temperatures, respectively. As the cavities interior surface of the copper insert was designed to be normal to the electric field of the mode of interest, we expected Ohmic losses due to induced currents in the normal metal to be minimized, with internal $\mathrm{Q}$ of the hybrid cavity being substantially higher than one for the $\mathrm{Cu}$ cavity.

To experimentally determine the internal Q we measured in reflection from one port of the cavity, with the second port closed with Al tape. We adjusted the coupling of the port to obtain an external quality factor close to the value of the expected internal Q. Our first low temperature measurement of the hybrid cavity yielded $Q \sim 15,000$, only a moderate improvement compared to $\mathrm{Cu}$ cavity. We attributed this behavior to small gaps between the superconducting and normal-metal interface of the cavity arising from the insert due to the manufacturing process (order of $\mu \mathrm{m}$ ) and to roughness of the copper surface. Recent results also demonstrated that interfaces between different parts of the 3D cavities play a crucial role in defining their quality factors [7].

To better thermalize our qubit to the dilution refrigerator, the normal metal copper is in contact with the chip. The chip is placed between the two halves of the cavity, where one side of the chip faces the copper, and the other Al. While beneficial for thermalizing the qubit, this specific design can incur more losses due to an air gap between some parts of the cavity.

To avoid losses in potential gaps in any mating faces, we deposited $\sim 2 \mu \mathrm{m}$ of $\mathrm{Al}$ on all $\mathrm{Cu}$ surfaces which are in contact with the $\mathrm{Al}$ cavity (the copper insert after evaporation of $\mathrm{Al}$ and other parts are shown in Figure 1(d)). Before deposition the surface of the copper inserts facing the interior of the cavity was covered with $\mathrm{Al}$ tape. Deposition was done in e-beam evaporator at a $45^{\circ}$ angle to taped surface under constant rotation of the insert about the deposition direction. After that procedure, and sealing the cavities mating faces with indium wire to ensure light tightness, the cavity demonstrated a $Q=102,000$, which is an order of magnitude improvement over the quality factor of the bare copper cavity. An additional measurement of the same cavity several months later showed a decrease of internal Q to the value of 80,000, which we associated with oxidation of the evaporated Al film. The quality factor of the cavity showed no power dependence within a wide range of powers from approx. $-50 \mathrm{dBm}$ to $-160 \mathrm{dBm}\left(\sim 10^{9}-10^{-2}\right.$ photons in the cavity).

The power-independence of the internal $\mathrm{Q}$ in a wide range suggests that two-level fluctuators do not play a dominant role in dissipation. The high internal quality factor of the cavity fully made of $\mathrm{Al}$ also proves that losses in a superconducting part are negligible. We, therefore, suggest that the losses are dominated by Ohmic losses in the copper insert especially along the interfaces with superconducting parts.

To demonstrate individual control of three superconducting qubits with an external magnetic field we used three transmon type qubits. The qubits were fabricated on an in- 
trinsic Si substrate of size $10 \mathrm{~mm}$ by $5 \mathrm{~mm}$, with a thickness of $500 \mu \mathrm{m}$ (see Figure 1(c)) in a single step of electron beam lithography followed by shadow evaporation of two Al layers of $20 \mathrm{~nm}$ and $40 \mathrm{~nm}$ thick with an oxidation step between the depositions. Each qubit consisted of two planar capacitor plates $700 \mu \mathrm{m}$ wide by $350 \mu \mathrm{m}$ tall. The plates were separated by $50 \mu \mathrm{m}$ and connected via a line interrupted by a micron size DC SQUID, playing the role of a magnetically tunable Josephson junction. The chip was placed in between the two halves of the cavity and magnetic flux supplied by three superconducting coils (two smaller and one larger) attached to the copper cavity were used to individually control the transition frequencies of all three qubits. The coils had 4,000 turns of $25 \mu \mathrm{m}$ superconducting wire and their diameters were $6 \mathrm{~mm}$ and $2 \mathrm{~mm}$ for large and small coils, respectively. The coil setup is virtually identical to one used to control three superconducting qubits coupled to a coplanar waveguide cavity on a chip [12].

The input and output ports of the cavity were then coupled asymmetrically for measuring qubit spectroscopy in transmission, with corresponding external quality factors of $Q_{\text {in }} \simeq 50,000$ and $Q_{\text {out }} \simeq 4,000$ (the output coupling was substantially increased to also perform time domain characterization of the qubits, data is not presented here). The fundamental mode of the cavity was shifted down to $7.295 \mathrm{GHz}$ due to the insertion of the chip. A typical spectrum of all of the qubits as function of the electrical current applied to one of the coils is shown in Figure 2(a). As the distance between qubits is small compared to the size of the magnetic coils, any current applied through one coil affects all qubits, as can be seen in Figure 2(a). The mutual inductances between the coils and qubits' SQUID loops can be determined by fitting the spectrum to the expected theoretical function: $h f_{i}=\left(E_{\operatorname{Imax}, i}\left|\cos \left(\pi \Phi_{i} / \Phi_{0}\right)\right| E_{c}\right)^{1 / 2}-E_{c}$, where $\Phi_{i}=\Phi_{\text {offset }, i}+\sum_{j} M_{i j} I_{j}$ and $\Phi_{0}$ is the magnetic flux quantum. The charging energy of the transmon is fixed by the geometry of capacitor pads and was chosen to be $E_{c} / h=130 \mathrm{MHz}$. The maximum Josephson energy of the SQUID at zero magnetic field $E_{\text {Jmax }, i}$, the flux offset $\Phi_{\text {offset }, i}$ and the mutual in-
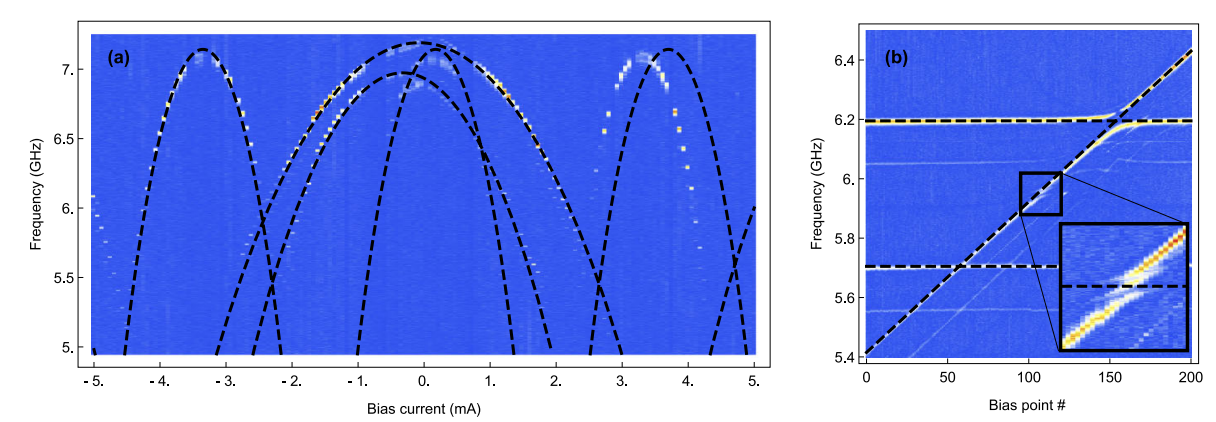

Figure 2 Three qubit spectroscopy. (a) Spectroscopy of the three transmon qubits. The $x$ axis indicates the electrical current value driven through the larger magnetic coil mounted to the cavity. The $y$ axis shows the frequency of the excitation tone sent to the input of the cavity together with the measurement tone. The color indicates transmission of the measurement tone through the cavity at its resonance frequency, recalibrated at each bias current with no excitation. The background transmission was subtracted for each bias current for clarity. The dashed black curves indicate a fit to the expected magnetic dependencies of the transmon frequencies allowing extraction of the mutual inductances between the coil and SQUID loops for each qubit. (b) Once the mutual inductance matrix between the coils and the qubits is extracted one can realize individual control of the qubit frequencies. In this example two of the qubits are kept at the chosen frequencies of 5.705 and $6.195 \mathrm{GHz}$, respectively, while the third qubit was made to change its frequency linearly with the bias parameters. The dashed black lines are shown as a guide for the eye. The inset shows a zoom in on the avoided level crossing observed around $(5.705+6.195) / 2=5.950 \mathrm{GHz}$ (the frequency is indicated by a dashed line in the inset). 
ductances $M_{i j}$ are the determined by the fitting the modulation of the frequency of qubit $i$ when current is applied to coil $j$. In the case that the coupling of the coils to different qubits are sufficiently different, the matrix can then be diagonalized, inverted and used for arbitrary control of the qubits frequencies. To demonstrate this, we fixed the frequencies of two qubits at $5.705 \mathrm{GHz}$ and $6.195 \mathrm{GHz}$ and tuned the frequency of the third qubit linearly (see Figure 2(b)). Figure 2(a) shows that the frequency modulations of the qubits are not perfectly periodic, most probably from the rearrangement of pinned magnetic vortices when changing external magnetic flux. Nonetheless, with fine adjustments of the mutual inductance matrix, it is always possible to achieve precise frequency control for all qubits.

This individual control over the qubits allowed us, for example, to see interesting spectrum features with clarity. In particular, an exchange interaction between the qubits mediated by resonator photons, manifested in the avoided level crossings of the qubit spectral lines [13], can be clearly seen for the third qubit at $6.195 \mathrm{GHz}$. Within the avoided level crossing, the eigenstates of the system are superpositions of the ground and excited states of the bare (non-interacting) qubits. The top spectral line of the avoided level crossing at $\sim 6.195 \mathrm{GHz}$ shows a clear gap corresponding to the anti-symmetric dark state which cannot be excited due to its symmetry [14].

It is interesting to note that in addition to the well-known features of the spectrum described, there are also some spectrum particularities not described by the conventional models. More specifically while the avoided level crossing at $6.195 \mathrm{GHz}$ is clearly visible, there is no observable avoided level crossing for another pair of qubits at $5.705 \mathrm{GHz}$. This phenomenon cannot be accounted for by the Tavis-Cummings Hamiltonian, describing several qubits interacting to a single quantized mode of electromagnetic field [15]. We speculate that the coupling through the resonator is compensated by the direct dipoledipole interaction between the closest qubits which gives rise to the exchange interaction of opposite sign. Similar behavior has been observed but not reported for the system of three qubits [16] but has never been studied in details.

In addition, a smaller avoided level crossing of the third qubit was observed half-way between the frequencies of the first and second qubits at $\sim 5.950 \mathrm{GHz}$. From the frequency matching conditions it may be attributed the process where two excitations of the third qubit are exchanged with excitations of the first and second qubits. This process cannot be explained neither by the Tavis-Cummings model nor by inclusion of the direct dipoledipole coupling. There is also a more complex higher order transition pattern that can also be seen on the background of the spectrum as faint lines. These complex peculiarities of the spectrum may be subject of further investigation using 3D hybrid cavities with flux control.

\section{Conclusions}

In this work we presented a new type of hybrid 3D cavity which can reach internal quality factors an order of magnitude greater compared to pure normal metal cavities without loosing the ability to use magnetic flux as a control parameter. The higher internal Q can offer longer life-times of the magnetically tunable qubits within 3D circuit QED architecture. The losses are dominated by the Ohmic losses in along the mating surfaces of the normal metal insert. Recently developed method for 3D printing [17] can possibly allow to manufacturing seamless hybrid cavity with higher internal Qs. In addition decreasing internal photon losses while maintaining the strong coupling of the cavity mode to the 
external circuitry is a necessary condition for the quantum networking with microwave photons. Finally, a cavity with both the large internal $Q$ and the magnetically control may find vast applications in experiments with hybrid quantum systems.

\section{Competing interests}

The authors declare that they have no competing interests.

\section{Authors' contributions}

Y. Reshitnyk designed the cavity. Y. Reshitnyk and M. Jerger performed the measurements. A. Fedorov and M. Jerger wrote the manuscript. A. Fedorov supervised the project. All authors read and approved the final manuscript.

\section{Acknowledgements}

We thank Prof. Y. Nakamura for providing us with a three-qubit sample and Prof. A. Wallraff for providing the design of the precision coils. We also thank Dr. V. Monarkha for helping us with measurement of power dependence of the quality factor of the cavity. M.J., A.F. were supported by the Australian Research Council Centre of Excellence CE1 10001013. Y.R. was supported by the Discovery Project DP150101033. AF was supported in part by the ARC Future Fellowship FT140100338.

Received: 30 May 2016 Accepted: 5 October 2016 Published online: 18 October 2016

\section{References}

1. Paik H, Schuster DI, Bishop LS, Kirchmair G, Catelani G, Sears AP, Johnson BR, Reagor MJ, Frunzio L, Glazman LI, Girvin SM, Devoret MH, Schoelkopf RJ. Observation of high coherence in Josephson junction qubits measured in a three-dimensional circuit QED architecture. Phys Rev Lett. 2011;107:240501. doi:10.1103/PhysRevLett.107.240501.

2. Weber SJ, Chantasri A, Dressel J, Jordan AN, Murch KW, Siddiqi I. Mapping the optimal route between two quantum states. Nature. 2014:511(7511):570-3.

3. Heeres RW, Vlastakis B, Holland E, Krastanov S, Albert WV, Frunzio L, Jiang L, Schoelkopf RJ. Cavity state manipulation using photon-number selective phase gates. Phys Rev Lett. 2015;115:137002. doi:10.1103/PhysRevLett.115.137002.

4. Probst S, Tkalčec A, Rotzinger H, Rieger D, Le Floch J-M, Goryachev M, Tobar ME, Ustinov AV, Bushev PA. Three-dimensional cavity quantum electrodynamics with a rare-earth spin ensemble. Phys Rev B. 2014;90:100404. doi:10.1103/PhysRevB.90.100404.

5. Tabuchi Y, Ishino S, Noguchi A, Ishikawa T, Yamazaki R, Usami K, Nakamura Y. Coherent coupling between a ferromagnetic magnon and a superconducting qubit. Science. 2015;349(6246):405-8. doi:10.1126/science.aaa3693. http://science.sciencemag.org/content/349/6246/405.full.pdf.

6. Angerer A, Astner T, Wirtitsch D, Sumiya H, Onoda S, Isoya J, Putz S, Majer J. Collective strong coupling with homogeneous Rabi frequencies using a 3D lumped element microwave resonator. Appl Phys Lett. 2016;109:033508. doi:10.1063/1.4959095

7. Wang C, Axline C, Gao YY, Brecht T, Chu Y, Frunzio L, Devoret MH, Schoelkopf RJ. Surface participation and dielectric loss in superconducting qubits. Appl Phys Lett. 2015;107:162601. doi:10.1063/1.4934486.

8. Reagor M, Paik H, Catelani G, Sun L, Axline C, Holland E, Pop IM, Masluk NA, Brecht T, Frunzio L, Devoret MH, Glazman $\mathrm{L}$, Schoelkopf RJ. Reaching 10 ms single photon lifetimes for superconducting aluminum cavities. Appl Phys Lett. 2013;102:192604. doi:10.1063/1.4807015.

9. Rigetti C, Gambetta JM, Poletto S, Plourde BLT, Chow JM, Córcoles AD, Smolin JA, Merkel ST, Rozen JR, Keefe GA, Rothwell MB, Ketchen MB, Steffen M. Superconducting qubit in a waveguide cavity with a coherence time approaching 0.1 ms. Phys Rev B. 2012;86(10):100506.

10. Bogorin DF, Ware M, MCClure DT, Sorokanich S, Plourde BLT. Reducing surface loss in 3D microwave copper cavities for superconducting transmon qubits. In: 2013 IEEE 14th international superconductive electronics conference (ISEC). 2013. p. 1-3. doi:10.1109/ISEC.2013.6604283.

11. Juliusson K, Bernon S, Zhou X, Schmitt V, le Sueur H, Bertet P, Vion D, Mirahimi M, Rouchon P, Esteve D. Manipulating Fock states of a harmonic oscillator while preserving its linearity. arXiv:1607.05204. (2016).

12. Fink JM, Bianchetti R, Baur M, Göppl M, Steffen L, Filipp S, Leek PJ, Blais A, Wallraff A. Dressed collective qubit states and the Tavis-Cummings model in circuit QED. Phys Rev Lett. 2009;103(8):083601. doi:10.1103/PhysRevLett.103.083601.

13. Majer J, Chow JM, Gambetta JM, Koch J, Johnson BR, Schreier JA, Frunzio L, Schuster DI, Houck AA, Wallraff A, Blais A, Devoret MH, Girvin SM, Schoelkopf RJ. Coupling superconducting qubits via a cavity bus. Nature. 2007:449(7161):443-7. doi:10.1038/nature06184.

14. Filipp S, van Loo AF, Baur M, Steffen L, Wallraff A. Preparation of subradiant states using local qubit control in circuit QED. Phys Rev A. 2011;84:061805. doi:10.1103/PhysRevA.84.061805

15. Tavis M, Cummings FW. Exact solution for an N-molecule-radiation-field Hamiltonian. Phys Rev. 1968;170(2):379-84. doi:10.1103/PhysRev.170.379.

16. Baur M, Fedorov A, Steffen L, Filipp S, da Silva MP, Wallraff A. Benchmarking a quantum teleportation protocol in superconducting circuits using tomography and an entanglement witness. Phys Rev Lett. 2012;108:040502. doi:10.1103/PhysRevLett.108.040502.

17. Creedon DL, Goryachev M, Kostylev N, Sercombe TB, Tobar ME. A 3D printed superconducting aluminium microwave cavity. Appl Phys Lett. 2016;109:032601. doi:10.1063/1.4958684 